\title{
Synergistic antitumor activity of pan- PI3K inhibition and immune checkpoint blockade in bladder cancer
}

\author{
Shaoming Zhu, ${ }^{1,2}$ A-Hong Ma, ${ }^{3}$ Zheng Zhu, ${ }^{4}$ Elio Adib (1),${ }^{4}$ Ting Rao, ${ }^{1,2} \mathrm{Na} \mathrm{Li},{ }^{1,5}$ \\ Kaiyuan $\mathrm{Ni}^{6}{ }^{6}$ Veera Chandra Sekhar Reddy Chittepu, ${ }^{4}$ Rao Prabhala, ${ }^{7}$ \\ Juan Garisto Risco, ${ }^{8}$ David Kwiatkowski, ${ }^{9}$ Kent Mouw, ${ }^{7}$ Guru Sonpavde (1) , 7 \\ Fan Cheng, ${ }^{2}$ Chong-xian Pan (i) ${ }^{1,4,10}$
}

To cite: Zhu S, Ma A-H, Zhu Z, et al. Synergistic antitumor activity of pan-PI3K inhibition and immune checkpoint blockade in bladder cancer. Journal for ImmunoTherapy of Cancer 2021;9:e002917. doi:10.1136/jitc-2021-002917

- Additional supplemental material is published online only. To view, please visit the journal online (http://dx.doi.org/10. 1136/jitc-2021-002917).

SZ, AM and ZZ contributed equally.

Accepted 31 August 2021
Check for updates

(c) Author(s) (or their employer(s)) 2021. Re-use permitted under CC BY-NC. No commercial re-use. See rights and permissions. Published by BMJ.

For numbered affiliations see end of article.

\section{Correspondence to \\ Dr Chong-xian Pan; chongxian_pan@hms.harvard. edu}

Dr Fan Cheng; urology1969@163.com

\section{ABSTRACT}

Background Immune checkpoint blockade (ICB) induces durable response in approximately $20 \%$ of patients with advanced bladder urothelial cancer (aUC). Over $50 \%$ of aUCs harbor genomic alterations along the phosphoinositide 3-kinase (PI3K) pathway. The goal of this project was to determine the synergistic effects and mechanisms of action of PI3K inhibition and ICB combination in aUC.

Methods Alterations affecting the PI3K pathway were examined in The Cancer Genome Atlas (TCGA) and the Cancer Dependency Map databases. Human and mouse cells with Pten deletion were used for in vitro studies. C57BL/6 mice carrying syngeneic tumors were used to determine in vivo activity, mechanisms of action and secondary resistance of pan-PI3K inhibition, ICB and combination.

Results Alterations along the PI3K pathway occurred in $57 \%$ of aUCs in TCGA. CRISPR (clustered regularly interspaced short palindromic repeats) knockout of PIK3CA induced pronounced inhibition of cell proliferation $(p=0.0046)$. PI3K inhibition suppressed cancer cell growth, migration and colony formation in vitro. PanPI3K inhibition, antiprogrammed death 1 (aPD1) therapy and combination improved the overall survival (OS) of syngeneic mice with PTEN-deleted tumors from 27 days of the control to 48, 37, and 65 days, respectively. In mice with tumors not containing a PI3K pathway alteration, OS was prolonged by the combination but not single treatments. Pan-PI3K inhibition significantly upregulated CD80, CD86, MHC-I, and MHC-II in dendritic cells, and downregulated the transforming growth factor beta pathway with a false discovery rate-adjusted q value of 0.001 . Interferon alpha response was significantly upregulated with aPD1 therapy (q value: $<0.001$ ) and combination (q value: 0.027 ). Compared with the control, combination treatment increased $\mathrm{CD}^{+}{ }^{+} \mathrm{T}$-cell infiltration $(p=0.005)$, decreased $T_{\text {reg }}$-cell infiltration $(p=0.036)$, and upregulated the expression of multiple immunostimulatory cytokines and granzyme $B(p<0.01)$. Secondary resistance was associated with upregulation of the mammalian target of rapamycin (mTOR) pathway and multiple Sprr family genes.

Conclusions The combination Pan-PI3K inhibition and ICB has significant antitumor effects in aUC with or without activated PI3K pathway and warrants further clinical investigation. This combination creates an immunostimulatory tumor milieu. Secondary resistance is associated with upregulation of the mTOR pathway and Sprr family genes.

\section{INTRODUCTION}

Bladder cancer is the 12th most common malignancy worldwide in 2018. ${ }^{1}$ There are more than 500000 new cases and above 200000 deaths every year. $^{2}$ In 2021, about 83730 new cases will be diagnosed with 17200 deaths in the USA. ${ }^{3}$ Approximately $90 \%$ of bladder cancers are urothelial carcinoma (UC). Even though most UCs are diagnosed at the non-muscle-invasive stage, it is metastatic UC that causes most of the mortalities. Currently, the first-line therapy for metastatic UC is a platinum-based combination therapy, such as gemcitabine plus cisplatin/carboplatin (GC) or methotrexate, vinblastine, doxorubicin/adriamycin and cisplatin (MVAC), which is highly toxic and rarely induces durable response. ${ }^{4}$ If patients do not have disease progression after the first-line chemotherapy, immunotherapy with avelumab maintenance is approved by the US Food and Drug Administration (FDA). ${ }^{5}$ If patients are cisplatin-ineligible or have disease progression after platinum-based chemotherapy, immunotherapy with an antiprogrammed cell death 1 (PD1) or PD ligand 1 (PD-L1) antibody is usually prescribed. ${ }^{6}$ The overall response rate of the five approved anti-programmed death 1 (aPD1)/PD-L1 antibodies is disappointingly around $20 \%$. In addition, approximately $15 \%$ of patients develop severe to life-threatening immunemediated toxicity. Other treatments at the salvage setting include a fibroblast growth factor receptor (FGFR) inhibitor erdafitinib and antibody-drug conjugate enfortumab vedotin and sacituzumab. ${ }^{7-9}$ These agents 
provide incremental benefits, are not curative and have substantial toxicities, for example, enfortumab vedotin (neurotoxicity, rash) and erdafitinib (mucositis, rash, visual toxicity).

With recent developments of deep sequencing and bioinformatics, it was found that most UCs harbor genomic alterations that can potentially be targeted for therapeutic applications. ${ }^{10}$ Among those alterations, over half of the UCs have alterations in the phosphoinositide 3-kinase (PI3K) /AKT/mammalian target of rapamycin (mTOR) pathway (online supplemental SI-3B) compared with less than $20 \%$ with FGFR2 or FGFR3 activating alterations that erdafitinib targets. PI3K is the downstream signal transducer of many cell surface receptors and plays important roles in cell survival, growth, proliferation, differentiation and metabolism. Abnormal activation of this pathway is often related to tumorigenesis and tumor progression. ${ }^{11}$ Of the four classes of PI3K, class I PI3K is the main subtype that activates AKT (also known as protein kinase B) signaling, and occurs as $\alpha, \beta, \delta$, and $\gamma$ isoforms. The $\alpha$ and $\beta$ isoforms are mainly expressed in tumor cells, while $\delta$ and $\gamma$ are mainly expressed in immune cells. Therefore, the PI3K signaling not only affects tumor cells but also plays important roles in immune response. T cell-specific deletion of PIK3CA has enhanced cytokine production and antitumor response. ${ }^{12}$ Ex vivo inhibition of PI3K- $\delta$ enhances therapeutic efficacy and memory of tumorspecific CD8 ${ }^{+} \mathrm{T}$ cells. ${ }^{13}$ Pan-PI3K inhibition enhances inflammatory response of dendritic cells (DCs). ${ }^{14}$ Here we studied that activity of a pan-PI3K inhibitor copanlisib with the IC50s of $0.5,3.7,6.4$, and $0.7 \mathrm{nmol} / \mathrm{L}$ against the $\alpha, \beta, \delta$, and $\gamma$ isoforms, respectively. ${ }^{15}$ The advantage of studying copanlisib is that it has already been approved by the FDA and, hence, can be relatively easy to be repurposed for other indications.

Even though immunotherapy has low response rate and high toxicity, one unique advantage is that some patients can achieve long-term disease-free survival secondary to immune surveillance after activation. Because of the multifunctional properties of PI3K in tumorigenesis and in regulation of immune cell functions, this project was designed to determine whether inhibition of the PI3K pathway could potentiate immunotherapy and regulate anticancer immunity in addition to its direct antitumor activity. The studies were also designed to decipher the underlying mechanisms of action and study resistance mechanisms.

\section{MATERIALS AND METHODS}

The details of Materials and Methods can be found at online supplemental SI-1.

\section{Cell lines and reagents}

Human UC cell lines were purchased from ATCC (American Type Culture Collection) . UPPL1541 and BBN963 are two murine UC cell lines kindly provided by William Y Kim, MD, PhD, at University of North Carolina. ${ }^{16}$ Cell lines were maintained at the recommended culture mediums.

\section{In vivo efficacy study}

Two cell lines, UPPL1541 and MB49, were used for in vivo efficacy studies. UPPL1541 harbors a PTEN deletion, while MB49 does not have alteration along the PI3K pathway that can be targeted by copanlisib. Cancer cells were subcutaneously injected into the flanks of C57BL/ 6 mice. When the volume of the tumors reached $80-140 \mathrm{~mm}^{3} \quad$ (volume=length $\times$ width $\times$ width $\times 1 / 2$ ), mice were randomly divided into four groups: control (Ctrl), copanlisib (Copan), aPD1 antibody and copanlisib +aPD1 combination (combo) groups. The treatment schedule was the Ctrl group, $0.01 \mathrm{M} \mathrm{HCl}, 250 \mu \mathrm{L}$, intravenous, 2 days on/ 5 days off; the copan group, copanlisib, $12 \mathrm{mg} / \mathrm{kg}$ in $0.01 \mathrm{M} \mathrm{HCl}$, intravenous, 2 days on/ 5 days off; aPD1 group, aPD1 antibody, $200 \mu \mathrm{g}$, intraperitoneal, two times per week; combo group, copanlisib, $12 \mathrm{mg} / \mathrm{kg}$, intravenous, 2 days on/ 5 days off; and aPD1 antibody, $200 \mu \mathrm{g}$ intraperitoneal, two times per week. At 2 weeks of treatment, five to six mice were sacrificed in each group, and tumor samples were collected and considered as the responder (' $R$ ') groups, that is, Rcopan, RPD1 and Rcombo, respectively. These tumors were used for RNAseq, quantitative reverse transcription polymerase chain reaction (RT-PCR), flow cytometry analysis, western blot and other tests. The sequences of the primers are listed at online supplemental SI-2. The remaining mice continued treatment until the end point, when the tumor grew to $1500 \mathrm{~mm}^{3}$, or when the diameter of the ulcerations exceeded $1.5 \mathrm{~cm}$. At that time, mice were sacrificed and tumor samples were collected. Those tumors were referred as the non-responder ('NR') group, which was named as NRcopan $(\mathrm{n}=12)$, NRPD1 $(\mathrm{n}=10)$ and NRcombo $(n=13)$, respectively.

\section{Statistical analysis}

At least three independent experiments were performed for each analysis described in this article. Student t-test or one-way analysis of variance was used to compare continuous parametric data between two groups and multiple groups, respectively. Overall survival (OS) of mice was analyzed using Kaplan-Meier survival curve and log-rank test. Statistical analysis was performed by GraphPad Prism V.8 software and SPSS V.19.0.

\section{RESULTS}

\section{Frequent alterations along the PI3K/AKT/mTOR pathway in advanced UC}

First, we analyzed The Cancer Genome Atlas (TCGA) database with 413 bladder cancer samples to determine the prevalence of the alterations in the PI3K/AKT/mTOR pathway in advanced UC. Compared with only $22 \%$ of alterations at FGFR2 and FGFR3 with less than $20 \%$ putative drivers (online supplemental SI-3A) which erdafitinib targets, $56.6 \%$ of UCs have at least one alteration 
A

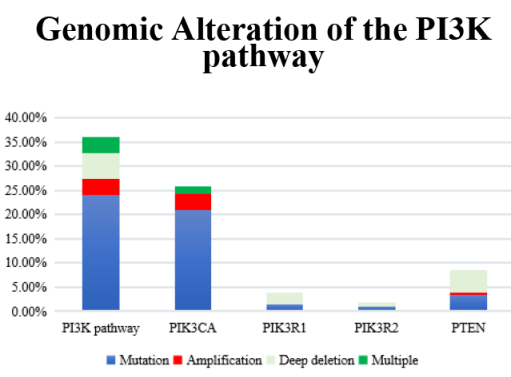

D

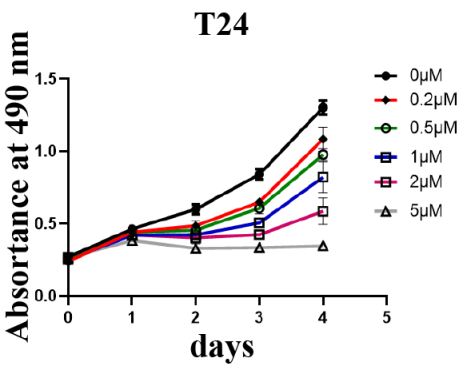

F

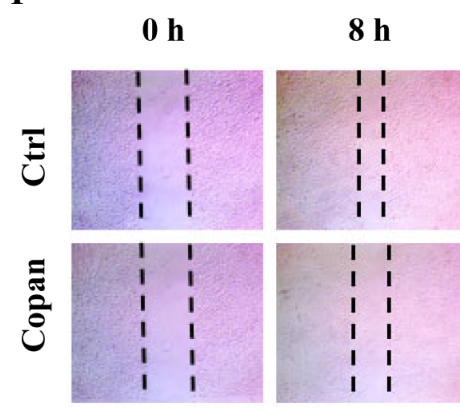

B

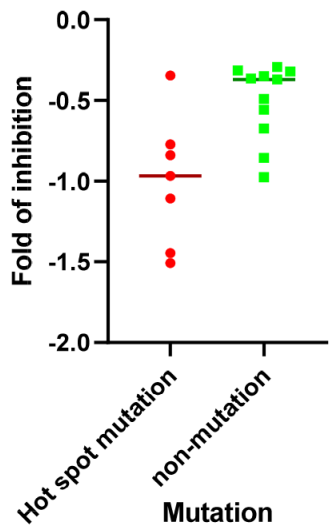

C.
Akt

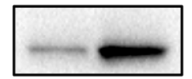

p-Akt

GAPDH
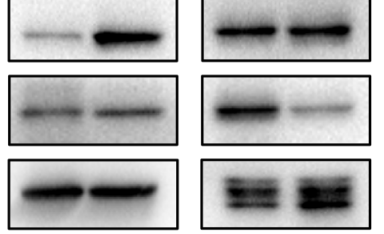

E
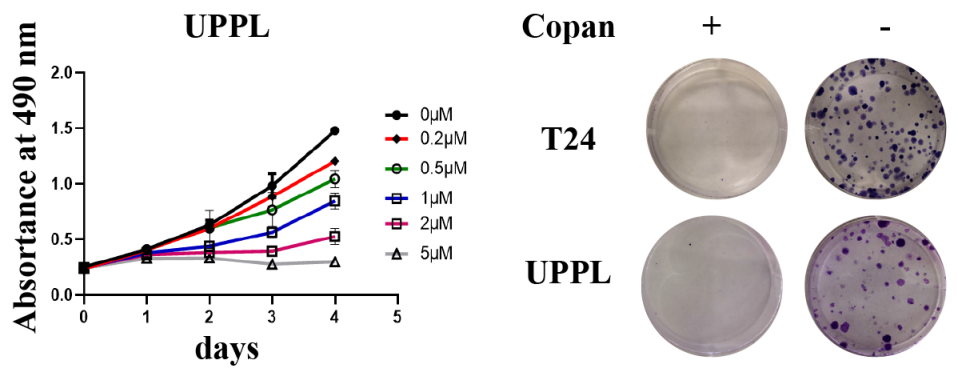

G

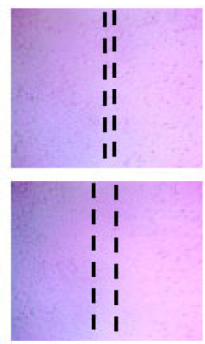

T24

UPPL

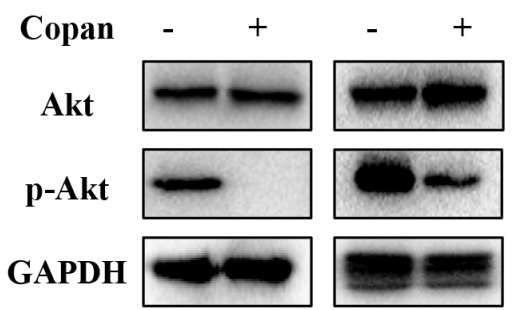

Figure 1 PI3K alteration and its effects on UC cells. (A) Genomic alterations that can be targeted by a PI3K inhibitor. The TCGA database was analyzed at the cBioPortal (www.cbioportal.org). Only genomic alterations of PIK3CA, PIK3R1, PIK3R2, and PTEN and combined alterations of these genes are shown, which can be targeted by copanlisib. At least one alteration occurs in $37 \%$ of UCs. (B) Effects of CRISPR knockout on bladder cancer growth. CRISPER knockout of PIK3CA induced significantly more inhibition of cell growth of 7 cell lines harboring PIK3CA activation mutations compared with 10 cell lines without PIK3CA mutations (log2 inhibition: -1.00 vs $-0.51, \mathrm{p}=0.0046$; depmap.org). (C) Activation of the PI3K signaling pathway by PTEN deletion. Western blots showed higher levels of p-Akt in the PTEN-deleted human T24 and mouse UPPL cells compared with wild-type 5637 and BBN cells. (D) Dose-dependent suppression of cell growth of T24 and UPPL cells by a panPI3K inhibitor copanlisib. (E) Inhibition of colony formation by copanlisib in T24 and UPPL cells. (F) Inhibition of wound healing of T24 cells. Cells were cultured for 24 hours before scratching. After scratching, cells were cultured with copanlisib at $1 \mu \mathrm{M}$. (G) Inhibition of the PI3K signaling by copanlisib. Western blot was performed of cells treated with copanlisib at $1 \mu \mathrm{M}$ for 12 hours. PI3K, phosphoinositide 3-kinase; TCGA, The Cancer Genome Atlas; UC, urothelial carcinoma, GAPDH, Glyceraldehyde 3-phosphate dehydrogenase.

along the PI3K pathway (online supplemental SI-3B,C). Among these mutations, $37 \%$ of mutations occur in one of the four genes (figure 1A), PIK3CA, PTEN, PIK3R1, and PIK3R2, which can be targeted by copanlisib, a medication already approved by the FDA for the treatment of follicular lymphoma. Alterations at PIK3CA are the most 
common and account for 26\% (105 samples) of all UC specimens.

Next, we analyzed the nature of the genetic alterations (figure 1A). As shown in online supplemental SI-3B,C, most of these alterations are putative drivers. For example, of the 105 cases with PIK3CA alterations (online supplemental SI-3B,D), 90\% (94 cases) are putative drivers, including 74 activation mutations, 14 gene amplifications and 6 combined alterations with activation mutation and gene amplification. PTEN is a negative regulator of the PI3K pathway. Correspondingly, of 35 alterations (8.6\%), 19 are deep deletion and 12 mutations that are known or likely to be oncogenic (online supplemental SI-3B,D), which, combined, account for $86 \%$ of all alterations on PTEN. Similar findings are observed with other genes along the PI3K pathway. We further analyzed the impact of PIK3CA expression on patient OS at the TCGA database. Since we previously showed that PIK3CA gene amplification could be a molecular driver, ${ }^{17}$ we hypothesized that high expression of PIK3CA could impact the UC outcomes. At the TCGA database, high expression is associated with inferior survival $(\mathrm{p}=0.044$, online supplemental SI-3E)

\section{Copanlisib effectively suppresses UC cell growth and migration through targeting PI3K/AKT signaling in vitro}

First, we looked into the Cancer Dependency Map database $^{18}$ and identified seven UC cell lines with PIK3CA activation mutations (online supplemental SI-4, highlighted). Overall, bladder cancer is sensitive to PI3KCA inhibition and at the top of the enriched lineages by CRISPR knockout $\left(\mathrm{p}=2.2 \times 10^{-5}\right)$. To determine the dependency of UC cells on the PI3K pathway, we analyzed and compared the inhibition of cell proliferation after CRISPR knockout of the PIK3CA gene. Ten bladder urothelial cell lines without any PIK3CA mutation were also sensitive to CRISPR knockout. However, significantly more inhibition of cell proliferation was observed in the seven cell lines with PIK3CA activation mutations (log2 inhibition: -1.00 vs -0.51 , Wilcoxon rank-sum, two-sided $\mathrm{p}=0.0046$; figure $1 \mathrm{~B})$.

We then determined whether blockade of the PI3K pathway could inhibit cell growth in cell lines. We previously showed that TCCSUP harbored a hotspot PIK3CA mutation E545K and was highly sensitive to PI3K inhibition. ${ }^{17}$ Here we tested additional human and mouse cell lines with mutations along the PI3K pathway. Human T24 harbors a Pten missense mutation, while murine UPPL has a Pten deletion. Both T24 and UPPL cells had high levels of phosphorylated AKT (p-Akt), a downstream target of PI3K activation, when compared with the control cells (figure 1C). Consistent with these findings, a pan-PI3K inhibitor copanlisib was effective in inhibiting cell growth, colony forming, and cell migration (figure 1D-F and online supplemental SI-5). Copanlisib treatment significantly decreased p-Akt levels in both cell lines (figure 1G).
We previously reported the inhibition of tumor growth in two patient-derived xenograft (PDX) models: one with a PIK3CA activation mutation and the other one with gene amplification. Both PDXs were highly sensitive to PI3K inhibitors. ${ }^{17} 19$ Taken together, all these data suggest that alterations along the PI3K pathway are putative drivers that can potentially be targeted for the treatment of UC.

\section{Pan-PI3K inhibition potentiated immune checkpoint blockade} (ICB) in syngeneic bladder cancer mouse models

Even though immunotherapy has been approved by the FDA for the treatment of metastatic UC, only a small proportion of patients achieve durable response, while cytotoxic chemotherapy and targeted therapy usually do not induce durable response. Hence, here we determined whether pan-PI3K inhibition could potentiate immunotherapy (figure 2A), and, if so, more patients could benefit from this combination. For this experiment, we implanted UPPL cells, which carry a PTEN deletion, in syngeneic immunocompetent C57BL/6 mice. Compared with the treatments with copanlisib and aPD1 antibody which only slightly delayed tumor growth, the combination treatment significantly delayed tumor growth (figure 2B). The median survivals of the control, copanlisib, aPD1 and combination groups were 27, 48, 37, and 65 days, respectively (figure 2C). When the control mice reached the end point (27th day of treatment), the tumors in the copanlisib and aPD1 groups were smaller than those in the control group $(\mathrm{p}<0.001$ and $\mathrm{p}=0.019$, respectively) but not significantly different between these two groups $(p>0.05)$ (figure 2D). Tumor volume in the combination group was the smallest when compared with the control group $(\mathrm{p}<0.001)$, copanlisib $(\mathrm{p}=0.001)$ and aPD1 $(p<0.001)$ monotherapy (figure 2D). There was no significant difference in mouse appearance and activities. The weight of the copanlisib and combination groups was slightly lower than that of the control and aPD1 groups but did not reach statistical significance (figure 2E).

To determine if this combination was also effective in tumors that do not have alteration along the PI3K pathway, we used another bladder cancer model MB49 in C57BL/6 mice. Copanlisib and aPD1 therapy, as a single agent, did not have any noticeable antitumor activity, while the combination significantly decreased tumor growth (figure 2F,G). The median survival of 17 days in the combination group was significantly longer than the control ( 11 days, $\mathrm{p}=0.0035)$ and copanlisib (11 days, $\mathrm{p}=0.018$ ) groups but longer, but not significant when compared with the aPD1 therapy ( 13 days, $\mathrm{p}=0.21$ ) (figure $2 \mathrm{H}$ ). When mice were euthanized, lungs were harvested for immunohistochemical (IHC) staining. The lungs from the control mice developed extensive metastatic cancer bilaterally. Copanlisib and aPD1 therapy slightly decreased lung metastasis, while little metastasis was observed in the lungs of the mice treated with the combination therapy (figure 2I).

In summary, the combination of copanlisib and aPD1 therapy could significantly suppress tumor growth and 


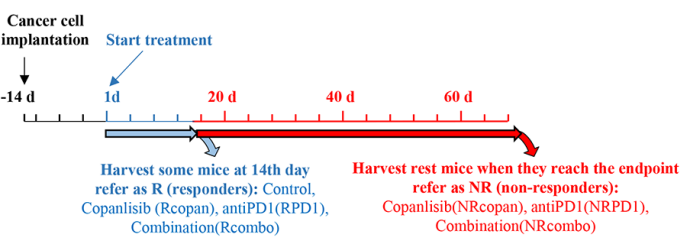

B
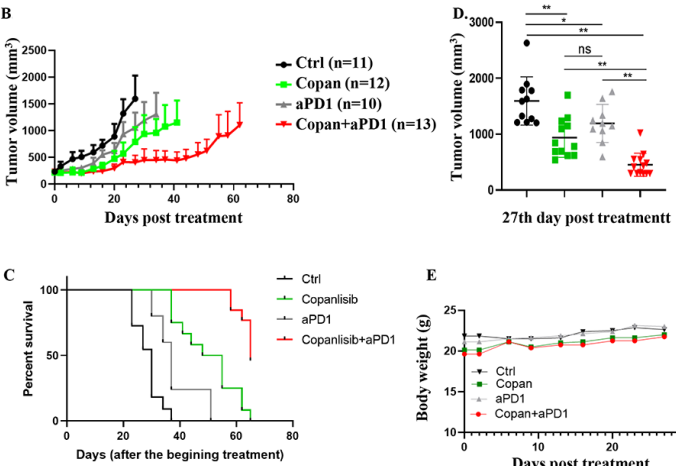

$\mathbf{F}$
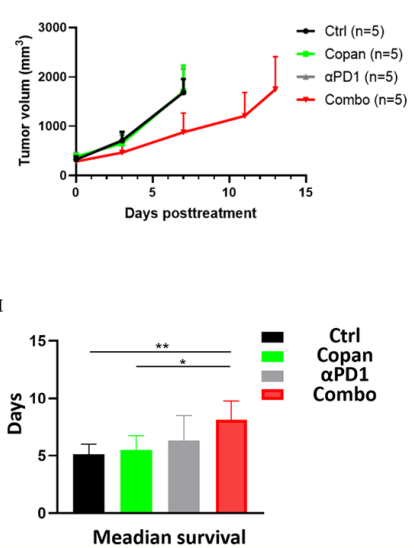

G
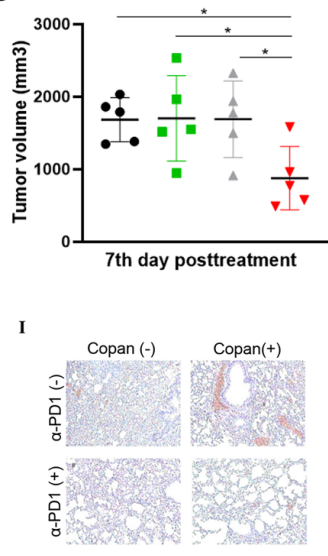

Figure 2 Synergistic antitumor effects of pan-PI3K inhibition and immune checkpoint blockade in vivo. (A) Experimental schema. C57BL/6 mice carrying syngeneic UPPL tumors were used for this experiment. $5 \times 10^{6}$ cells were injected subcutaneously at the flank around 2 weeks before treatments. When tumor sizes reached around $100 \mathrm{~mm}^{3}$, mice were randomized into the following four treatment groups at day 0: Ctrl group: $250 \mu \mathrm{L} 0.01 \mathrm{M} \mathrm{HCl}$ intravenously once per day, 2 days on $/ 5$ days off; copanlisib: $12 \mathrm{mg} / \mathrm{kg}$ caudal intravenous injection, 2 days on $/ 5$ days off; aPD1 antibody (Bioxcell, Cat\# BE0146, Lebanon, New Hampshire, USA), $200 \mu \mathrm{g}$ intraperitoneally injected two times per week and combination of copanlisib and aPD1 antibody. Mice were followed up for daily activity, weight and tumor size. Two weeks later, the tumors were still responding to the treatment (hence the responder or R groups); some mice were randomly sacrificed in each group; tumor samples were collected and named Ctrl, Rcopan, RPD1, and Rcombo, respectively. The end points included tumor sizes of $\geq 1500 \mathrm{~mm}^{3}$, ulcer diameter of $\geq 1.5 \mathrm{~cm}$ or body weight loss of $\geq 20 \%$. At that point, the mice were sacrificed, and tumor samples were collected and named as the 'NR' group. NRcopan $(n=12)$, NRPD1 $(n=10)$, and NRcombo $(n=13)$. (B) Tumor growth curve. (C) Overall survival. Compared with 27 days of the Ctrl group, the median survival of copanlisib, aPD1 therapy and combination was 48,37 and 65 days, respectively. (D) Tumor size. On the 27th day, the tumor sizes of mice in each group were measured and compared. (E) Body weight changes during treatment. $(\mathrm{F}-\mathrm{H})$ Tumor efficacy study with MB49 tumors. MB49 cells do not harbor activation alteration along the PI3K pathway. Mice carrying MB49 tumors were randomized into four groups: Ctrl, copanlisib, aPD1 and combination, and received the same treatments as described previously. Some tumors reached the humane study end point at day 7 in the Ctrl, copanlisib and aPD1 groups, while the mice in the combination group did not reach the end point until day 13 of treatment. (F) Tumor growth curve of MB49 tumors. (G) Tumor sizes measured at day 7. (H) Median survival. (I) Lung metastasis. The lung from the Ctrl mice contained extensive bilateral spontaneous metastasis. Copanlisib and aPD1 therapy slightly decreased lung metastasis, while little metastasis was visible in the lung from the combination group. ${ }^{*} \mathrm{P}<0.05$, ${ }^{\star \star} \mathrm{P}<0.01$. aPD1, antiprogrammed death 1; Ctrl, control; NR, non-responder; ns, not significant; PI3K, phosphoinositide 3-kinase.

prolong survival of bladder cancer models with or without activation alteration along the PI3K pathway.

\section{Treatments induced differential gene expression}

To decipher the underlying mechanisms of enhanced antitumor effects, we collected tumor tissues at baseline, 2 weeks after treatment and at the time of resistance, and performed RNA sequencing. The 2-week time point was chosen because tumors were still responding to treatments while mice had sufficient time to develop antitumor immune response. A 2-week course of treatment was associated with a significant upregulation (log-fold change $>2$, false discovery rate (FDR)-adjusted $p$ value $<0.05$; online supplemental SI-6, SI table 1) of 21 genes, and a significant downregulation (log-fold change $<-2$, FDR-adjusted $\mathrm{p}$ value $<0.05$ ) of 3 genes in the copanlisib group. The upregulation and downregulation genes were six and one, respectively, in the copanlisib plus aPD1 antibody combination group (online supplemental SI-6, SI table 1). There was no overlap between the two sets of differentially expressed genes.

We also determined how aPD1 therapies affected gene expression. The upregulated and downregulated genes associated with aPD1 treatment were 68 and 72 genes, respectively (log-fold change $<-2$, adjusted $p$ value $<0.05$; online supplemental SI-6, SI table 2). Two of these genes, CASP12 and ORM1, were upregulated in both the aPD1 $\left(\mathrm{p}=8.41 \times 10^{-5}\right.$ and $4.33 \times 10^{-4}$, respectively) and the combo $\left(\mathrm{p}=1.81 \times 10^{-6}\right.$ and $2.35 \times 10^{-5}$, respectively) groups. Caspase 12 , encoded by the CASP12 gene, belongs to the family of inflammatory caspases which process and activate inflammatory cytokines, such as interleukin (IL)-1 and 18. It mediates apoptosis induced by endoplasmic reticulum stress. ${ }^{20}{ }^{21}$ ORM1 is an acute-phase protein related to immune response even though its full functions are still not known. ${ }^{22}$ Hence, upregulation of these two genes in the aPD1 and combination treatment groups was likely physiologically relevant. 


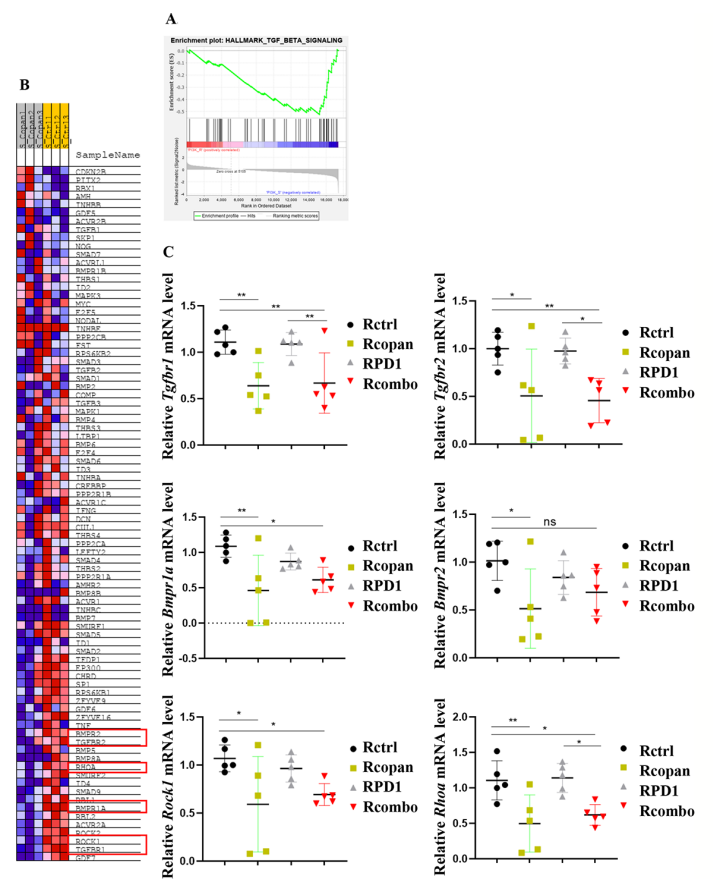

D
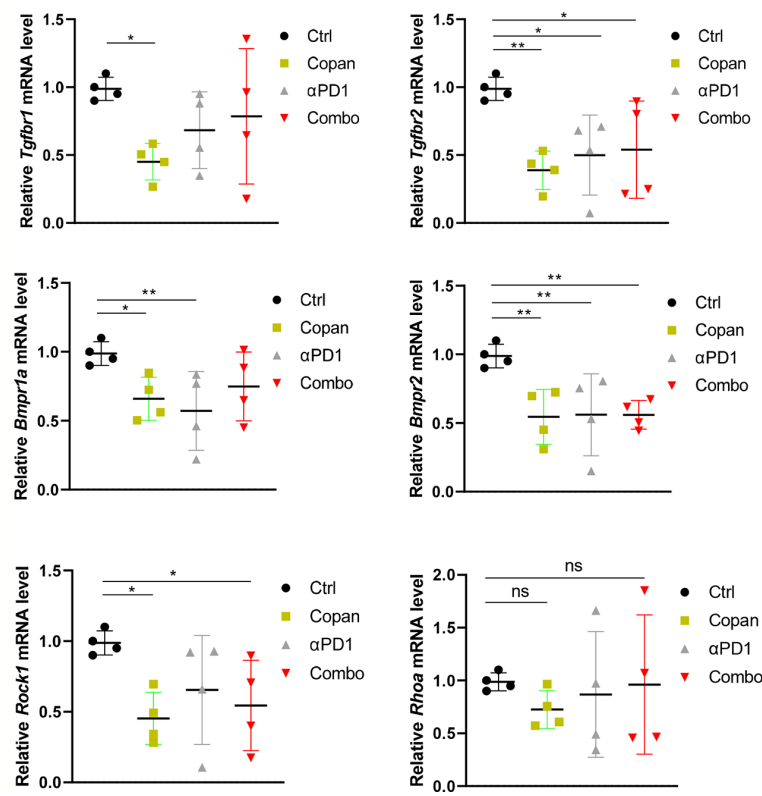

Figure 3 Downregulation of the TGF- $\beta$ pathway on treatment. (A) GSEA. Tumor tissues were harvested at the baseline (Ctrl) and 2 weeks after treatment started (responder or R groups) for transcriptome sequencing. GSEA was performed among pathways altered by treatment. Compared with Ctrl, treatment with copanlisib for 2 weeks significantly downregulated the TGF- $\beta$ pathway. NES: -1.70 and the FDR-adjusted $p$ value: 0.001 . (B) Heatmap showing alterations of expression of the TGF- $\beta$ pathway genes. (C) Quantitative RT-PCR of selected TGF- $\beta$ pathway genes of tumors collected after 2 weeks of treatment. (D) Quantitative RT-PCR of selected TGF- $\beta$ pathway genes of tumors collected after 3 days of treatment. ${ }^{*} P<0.05$, ${ }^{\star \star} P<0.01$. aPD1, antiprogrammed death 1; Ctrl, control; FDR, false discovery rate; GSEA, gene set enrichment analysis; NES, normalized enrichment score; ns, not significant; RT-PCR, reverse transcription PCR.

\section{PI3K inhibition downregulated the Transforming growth factor beta (TGF- $\beta$ ) pathway}

To study how PI3K inhibition potentiated anticancer immunity induced by aPD1 therapy, we performed gene set enrichment analysis (GSEA) of the RNASeq data after 2 weeks of treatment with copanlisib and found that the TGF- $\beta$ pathway was the top major pathway downregulated by copanlisib treatment (figure 3 and online supplemental SI table 3). The normalized enrichment score (NES) and FDR-adjusted $\mathrm{p}$ value were -1.7 and 0.001 , respectively (figure $3 \mathrm{~A}$ and online supplemental SI table $3)$. The TGF- $\beta$ pathway changes in the aPD1 (NES: -1.1 , FDR-adjusted p value: 0.583 ; online supplemental SI table 4) and the combination group (NES: -0.7, FDR-adjusted $\mathrm{p}$ value: 1.0; online supplemental SI table 5) were not significant.

We further confirmed the perturbation of the TGF- $\beta$ signaling pathway by quantitative RT-PCR of selected genes along the TGF- $\beta$ pathway. A heatmap showed clear downregulation of multiple genes along the pathway in the copanlisib group (figure 3B). Of these genes, we selected a few TGF- $\beta$ receptors (such as Tgfbr1, Tgfbr2, Bmprla and Bmpr2) and TGF- $\beta$ downstream genes Rock1 and Rhoa (figure 3C). Significant downregulation $(\mathrm{p}<0.05)$ was observed in all these genes in the specimens from mice treated with copanlisib. Except Bmpr2, significant downregulation $(\mathrm{p}<0.05)$ was observed in all other genes in the group treated with copanlisib and aPD1 combination. We did not observe any significant difference between the control and aPD1 groups. This suggests that pan-PI3K inhibition downregulated the immunosuppressive TGF- $\beta$ pathway and potentiated anticancer immunity.

At 2 weeks, the tumor growth curves had already widely separated and tumor microenvironment (TME) might have changed dramatically. To determine whether alterations of the TGF- $\beta$ pathway were secondary to the direct effects of copanlisib treatment, we harvested UPPL tumors at 3 days after copanlisib treatment and performed quantitative RT-PCR of genes along the TGF- $\beta$ pathway. Similar downregulation of the TGF- $\beta$ pathway genes was observed (figure 3D), suggesting that downregulation of the TGF- $\beta$ pathway was more likely secondary to the direct effect of PI3K inhibition rather than TME changes.

\section{Combination therapy remodeled tumor immune microenvironment}

Next, we determined how treatments affected immune cell infiltration at the TME. Two weeks after treatment started, fresh tumor specimens were harvested, singlecell populations prepared and flow cytometry performed to determine immune cell infiltration. The number of $\mathrm{CD}^{+} \mathrm{T}$ cells slightly increased but did not reach statistically significant level in the groups treated with copanlisib $(3.94 \% \pm 1.54 \%$,$) and aPD1 (3.24 \% \pm 1.47 \%)$ when compared with the control group $(2.1 \% \pm 1.37 \%$, figure 4 and online supplemental SI-7A). However, the number of 

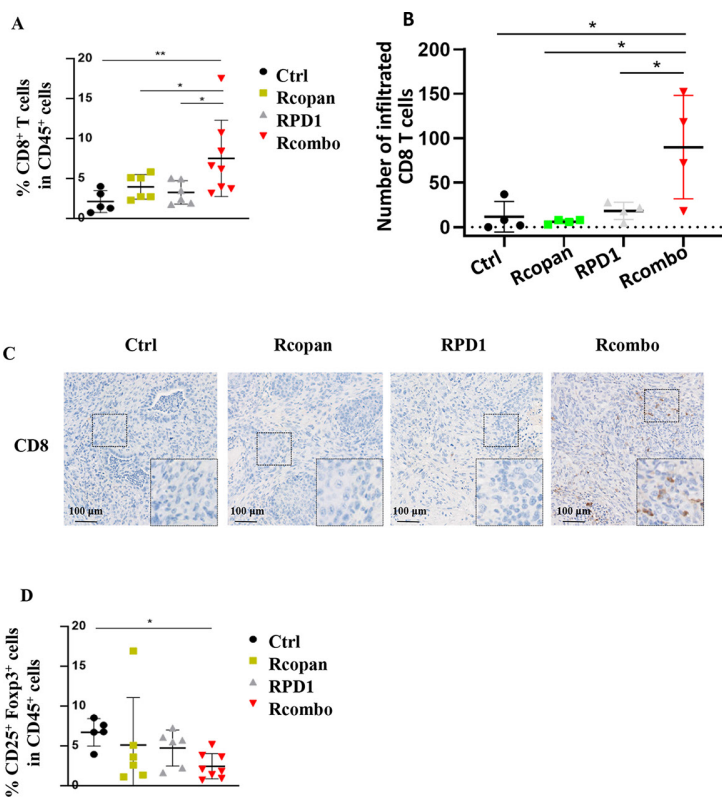
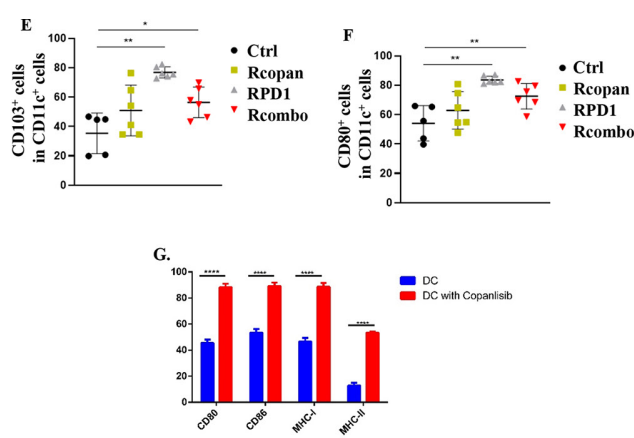

$\mathbf{H}$
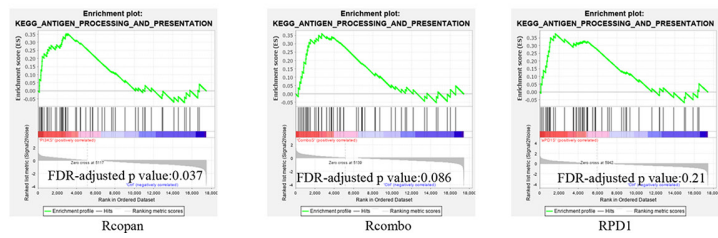

Figure 4 Alterations of immune cell infiltration at tumor microenvironment. (A) Infiltration of CD8 T cells determined by flow cytometry. Single cell suspensions were prepared from tumors harvested at 2 weeks after treatment, and flow cytometry was performed with anti-CD4 and anti-CD8 monoclonal antibodies. The CD8 ${ }^{+}$T-cell infiltration was $2.1 \% \pm 1.37 \%, 3.94 \% \pm 1.54 \%$, $3.24 \% \pm 1.47 \%$, and $7.52 \% \pm 4.78 \%$ for the Ctrl, copanlisib, aPD1, and combination treatment, respectively. The increase of $\mathrm{CD}^{+} \mathrm{T}$ cells in the combination treatment was significant when compared with the $\mathrm{Ctrl}(\mathrm{p}=0.005)$, copanlisib $(p=0.039)$ and aPD1 treatment $(p=0.016)$. The differences between copanlisib and Ctrl $(p=0.324)$, aPD1 and Ctrl $(p=0.538)$ and between copanlisib and aPD1 groups $(p=0.691)$ were not significant. There was no significant difference among CD4 ${ }^{+} \mathrm{T}$-cell infiltrations for these four treatments. (B,C) IHC staining of CD8 T cells in tumors 2 weeks after treatment. (D) Combination treatment significantly decreased CD25 $5^{+}$Foxp $3^{+} \mathrm{T}$ cells in CD45 ${ }^{+}$cells. Foxp $3+\mathrm{T}_{\text {reg }}$ cells were lower in the combination group when compared with the Ctrl $(p=0.036)$. The difference between combination and single treatments did not reach statistical significance. (E) Immunotherapy increased DCs at drainage lymph nodes. The proportions of CD103 ${ }^{+} \mathrm{CD}_{11 \mathrm{c}^{+}}$tissueresiding DCs in all three treatment groups were higher than those in the Ctrl group, but only those in the aPD1 ( $p<0.001)$ and combination $(p=0.011)$ groups reached a statistically significant difference. $(F)$ Immunotherapy induced expression of CD80 costimulatory molecules. CD80 $0^{+} \mathrm{CD} 11 \mathrm{c}^{+} \mathrm{DCs}$ were much higher in the aPD1 $(p<0.001)$ and combination $(p=0.006)$ groups when compared with the Ctrl. There was no difference between the copanlisib and Ctrl groups $(p=0.153)$. (G) Copanlisib upregulated CD80, CD86, MHC-I, and MHC-II of DCs. DC line DC2.4 cells were treated with copanlisib at $200 \mathrm{nM}$ for 48 hours before flow cytometry analyses. Compared with the Ctrl, copanlisib significantly upregulated the expression of $C D 80\left(p=4.36 \times 10^{-5}\right), C D 86$ $\left(p=8.79 \times 10^{-5}\right)$, MHC-I $\left(p=5.80 \times 10^{-5}\right)$ and MHC-II $\left(p=1.10 \times 10^{-4}\right) .(H)$ Upregulation of the antigen processing and presentation machinery. GSEA were performed with the RNAseq information of tumors at 2 weeks of treatment. The antigen processing and presentation machinery were significantly upregulated in the copanlisib group (NES: 1.65 and FDR p value=0.037, left panel), borderline in the combination group (NES: 1.52 and FDR p value=0.086, middle panel), but not in the aPD1 group (NES: 1.54 and FDR $p$ value $=0.21$, right panel). aPD1, antiprogrammed death 1; Ctrl, control; DC, dendritic cell; FDR, false discovery rate; GSEA, gene set enrichment analysis; NES, normalized enrichment score; ns, not significant.

$\mathrm{CD}^{+} \mathrm{T}$ cells was much higher in the combination group $(7.52 \% \pm 4.78 \%)$ compared with that in the control group $(\mathrm{p}=0.005)$, copanlisib-sensitive $(\mathrm{p}=0.039)$ and aPD1sensitive $(\mathrm{p}=0.016$; figure 4 and online supplemental SI-7A) groups. We used two other approaches to confirm that T-cell infiltration was induced in the treatment groups. First, we performed IHC staining and confirmed increased CD8 T-cell infiltration in the tumors of the combination group (figure 4B,C). We then performed Nanostring GeoMX Digital Spatial Profiler (DSP) analyses of the tumors and confirmed increase of $\mathrm{T}$ cells in the tumors of the combination group (online supplemental SI-8). In addition, several other immune activation markers on the panel, such as CD27, CD28, CD40, CD127 and ICOS, were also upregulated (online supplemental SI-8). $\mathrm{T}_{\text {reg }}$ cells are associated with immunosuppressive
TME by consuming proinflammatory cytokines (such as IL-2), releasing of anti-inflammatory cytokines (such as IL-6 and TGF- $\beta$ ), upregulating inhibitory immune checkpoints and recruiting immunosuppressive immune cells. ${ }^{23}$ Consistent with its functionality, $\mathrm{T}_{\text {reg }}$ cells in the combination group significantly decreased when compared with the control ( $\mathrm{p}=0.036$, figure $4 \mathrm{D}$ and online supplemental SI-7B). Compared with the control, $\mathrm{T}_{\text {reg }}$ cells in the copanlisib $(\mathrm{p}=0.435)$ and aPD1 $(\mathrm{p}=0.339)$ alone groups slightly decreased, but did not reach statistical significance. $\mathrm{CD} 4^{+} \mathrm{T}$ cells did not change significantly among the four groups (online supplemental SI-7A).

As antigen-presenting cells, DCs play critical roles in adaptive antitumor immunity. When damage-associated molecular patterns are released from stressed or dying cells, they activate DCs in tumor tissue and facilitate the 
phagocytosis of tumor-associated antigens. Activated DCs then migrate to tumor drainage lymph nodes and cross-present tumor-associated antigens to $\mathrm{T}$ cells. To determine how different treatments affected DCs in local drainage lymph nodes, we harvested those local lymph nodes, made single cell suspension and performed flow cytometry. The proportions of $\mathrm{CD}_{103^{+}}$tissue-resident DCs in CD11 $c^{+}$DCs in all three treatment groups were higher than that in the control group, but only those in the aPD1 $(p<0.001)$ and combination $(p=0.011)$ groups reached statistically significant difference (figure $4 \mathrm{E}$ and online supplemental SI-7C). The DCs in lymph nodes were borderline difference $(\mathrm{p}=0.051)$ between the control and copanlisib groups. During antigen presentation, DCs promote antigen presentation and T-cell activation by increasing the expression of costimulatory molecules CD80 (B7-1) and CD86 (B7-2). Similarly, CD80 ${ }^{+}$DCs were higher in all treatment groups but significantly higher in the aPD1 $(\mathrm{p}<0.001)$ and combination $(\mathrm{p}=0.006)$ groups when compared with the control (figure $4 \mathrm{~F}$ and online supplemental I-7C). The difference was not statistically different between the control and copanlisib groups ( $p=0.153)$. To further determine the effects of PI3K inhibition on DCs, mouse DC cell line DC2.4 was incubated with copanlisib for 48 hours. Compared with the control, copanlisib treatment significantly upregulated the expression of CD80 $\left(\mathrm{p}=4.36 \times 10^{-5}\right), \operatorname{CD} 86\left(\mathrm{p}=8.79 \times 10^{-5}\right)$, MHC-I $\left(\mathrm{p}=5.80 \times 10^{-5}\right)$ and MHC-II $\left(\mathrm{p}=1.10 \times 10^{-4}\right)$ on DC2.4 cells (figure 4G and online supplemental SI-9). Nanostring GeoMX analyses showed upregulation of CD40 in tumor specimens (online supplemental SI-8).

Next, we performed GSEA of the RNASeq data after 2 weeks of treatments. The antigen processing and presentation machinery was upregulated in the copanlisib treatment group with the NES and FDR-adjusted $p$ values of 1.65 and 0.037 (figure 4H, left panel). It was borderline upregulated in the combination group with the NES and FDR-adjusted $\mathrm{p}$ values of 1.52 and 0.086 , respectively (figure $4 \mathrm{H}$, middle panel). It was not significantly upregulated in the aPD1 treatment group with the FDR-adjusted $\mathrm{p}$ value of 0.21 (figure $4 \mathrm{H}$, right panel). These findings suggest that the upregulation of the antigen processing and presentation machinery was mainly secondary to the copanlisib treatment.

It has been suggested that PI3K $\gamma$ inhibition could overcome resistance to ICB through targeting myeloid cells. ${ }^{25}$ We analyzed the RNA expression through RNAseq and protein expression through Nanostring GeoMX DSP and did not find any significant change of myeloid cell marker expression, suggesting that, at least in the UPPL model studied here, myeloid cells might not be the main target of copanlisib in potentiating immune checkpoint inhibitor.

\section{Combination therapy stimulated proimmune cytokine production}

In the TME, many cytokines affect immune cell migration and function. Here we determined how the treatments affected cytokine production at the tumors. Type I interferons play critical roles in antitumor immunity. ${ }^{26}$ GSEA analyses showed that the interferon-alpha response was significantly upregulated with aPD1 treatment (NES: 3.45 and FDR-adjusted $\mathrm{p}$ value $<0.001$, figure $5 \mathrm{~A}$ and online supplemental SI table 4) and combination (NES 1.56 and FDR adjusted $p$ value $=0.027$, online supplemental SI table 5) treatments, but not in the copanlisib group (NES 0.97 and FDR adjusted $\mathrm{p}$ value $=0.606$, online supplemental SI table 3). The interferon-gamma response was also significantly upregulated with aPD1 treatment (NES: 2.93 and FDR-adjusted $\mathrm{p}$ value $<0.001$, figure $5 \mathrm{~B}$ and online supplemental SI table 4) but not in the copanlisib (NES -0.7 and FDR adjusted $p$ value $=1$, online supplemental SI table 3) and combination treatment (NES 1.29 and FDR adjusted $\mathrm{p}$ value $=0.147$, online supplemental SI table 5). Quantitative RT-PCR of Ifn-a1 and Ifn-a2 showed that, compared with the control, they significantly increased in the aPD1 $(\mathrm{p}<0.05)$ and combination $(\mathrm{p}<0.01)$ groups, and did not increase significantly in the copanlisib group ( $\mathrm{p}=0.677$ and 0.686 , respectively) (figure 5C,D). IL-6 and IL-10 are two cytokines involved as negative regulators of immune response. They were slightly decreased in the aPD1 and combination treatments, respectively (online supplemental SI-10).

Granzyme B (Gzmb) is a serine protease commonly secreted by NK and cytotoxic T cells to mediate apoptosis in target cells. Consistent with its function, Gzmb mRNA increased with treatments, especially in the groups treated with aPD1 (0.001) and combination therapies $(\mathrm{p}<0.001$, figure $5 \mathrm{E})$. There was no statistical difference between the control and copanlisib treatments $(\mathrm{p}=0.647)$. IHC staining confirmed the upregulation of GZMB protein in the combination group (figure $5 \mathrm{~F}, \mathrm{G}$ ). In addition, Nanostring GeoMX analyses showed the Gzmb protein was significantly upregulated in the combination treatment group (online supplemental SI-8).

\section{PI3K signaling pathway changed during the treatment course} We next determined how the PI3K signaling changed during treatment. At 2 weeks after treatment started, we did not observe any significant changes of gene expression along with the PI3K/AKT/mTOR pathway (NES: -0.9 , FDR $p$ value $=0.862$; online supplemental SI table 3 ) or the MTORC1 pathway (FDR $\mathrm{p}$ value $=1$, online supplemental SI-11 per GSEA). We confirmed that the total AKT level did not differ significantly among these four groups (figure 6A, left panel, top row). We then investigated the signaling activity of this pathway. While the aPD1 treatment did not significantly decrease p-Akt, the p-Akt levels in the copanlisib and combination groups significantly diminished when compared with the control or aPD1 groups $(\mathrm{p}<0.01$; figure $6 \mathrm{~A}$, right panel). These findings suggest that, while the gene expression levels, both at the mRNA and protein levels, did not alter significantly within such a short time (2 weeks) of treatment, the PI3K signaling activities were blocked by copanlisib and its combination with aPD1. 
A

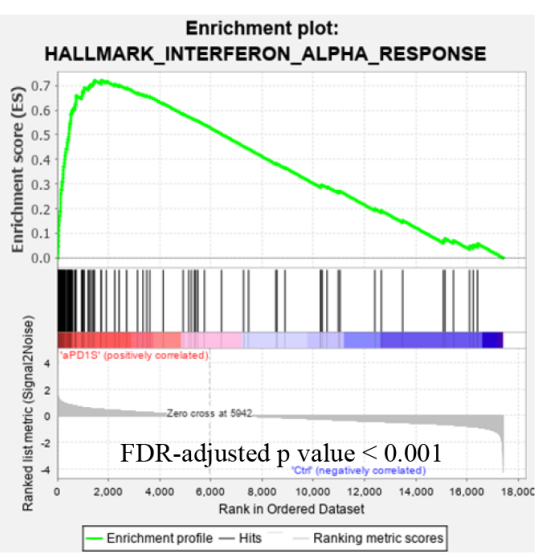

B.

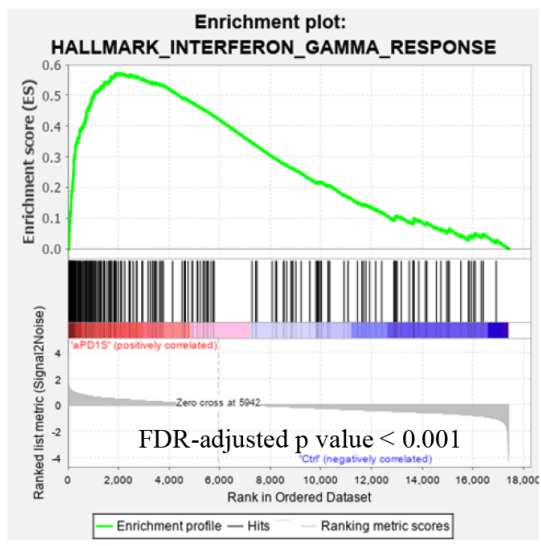

Figure 5 Upregulation of immunostimulatory cytokines with treatments. (A) Upregulation of interferon alpha response with aPD1 treatment. GSEA revealed NES: 3.45 and FDR-adjusted $p<0.001$. (B) Upregulation of interferon gamma with aPD1 treatment. GSEA revealed NES: 2.93 and FDR-adjusted p<0.001. (C,D) Quantitative RT-qPCR of Ifn-a1 and Ifn-a2. RT-PCR was performed to detect the mRNA expression level of cytokines in tumor tissues at 2 weeks after treatment. (E) Increase of Gzmb in the combination group as determined by quantitative RT-PCR. (F) IHC staining of Gzmb. (G) Quantitation of Gzmb IHC staining. Tumors harvested at 2 weeks after treatments were used for $\mathrm{IHC}$ staining. ${ }^{*} \mathrm{P}<0.05,{ }^{* *} \mathrm{p}<0.01$; five specimens in each group. aPD1, antiprogrammed death 1; Ctrl, control; FDR, false discovery rate; GSEA, gene set enrichment analysis; Gzmb, granzyme $\mathrm{B}$; NES, normalized enrichment score; RT-PCR, reverse transcription polymerase chain reaction.

Next, we performed GSEA to identify pathways that altered in the resistant tumors. The Akt/mTOR pathway was consistently upregulated in all three resistant groups when compared with the corresponding sensitive groups: copanlisib (NES: 1.61, FDR-adjusted $\mathrm{p}=0.015$; figure $6 \mathrm{~B}$ and online supplemental SI table 6), aPD1 therapy (NES: 1.60 , FDR-adjusted $\mathrm{p}=0.024$, online supplemental SI table 7 ) and combination (NES: 1.95, FDR-adjusted $\mathrm{p}=0.001$; online supplemental SI table 8).

To further confirm that the Akt/mTOR pathway was indeed upregulated in the resistant tumors, we selected three genes, S100A8, S100A9 and LCN2, that are related and coregulated with the Akt/mTOR pathway, and used
RT-qPCR to validate their upregulation (figure 6C). S100a9 and S100a8 are members of the S100 family of calcium-binding proteins, and stimulate the PI3K/Akt/ mTOR pathway. ${ }^{27}$ Lipocalin-2 (Lcn 2), also known as neutrophil gelatinase-associated lipocalin, is involved in inflammation, ${ }^{28}$ homeostasis, tumorigenesis and progression, and chemotherapy resistance. ${ }^{29}{ }^{30}$ High expression of $L c n 2$ is associated with inferior survival in UC (online supplemental SI-12A). RT-qPCR confirmed that the mRNA expression of these three genes in combination-resistant tumors was higher than that in sensitive tumors $(\mathrm{p}<0.01)$ and control $(\mathrm{p}<0.05)$ (figure 6D). Gene Multiple Association Network Integration Algorithm (GeneMANIA) 
A.

Sctrl Scopan SPD1 Scombo

Akt

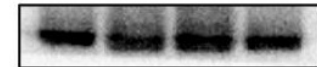

p-Akt

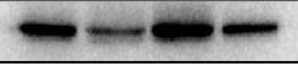

GAPDH

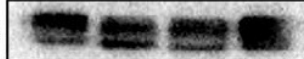

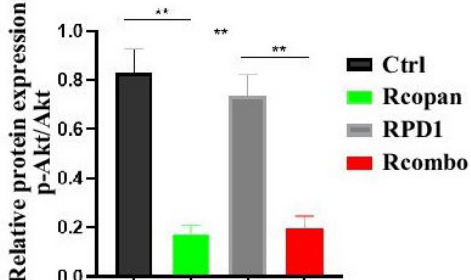

C.

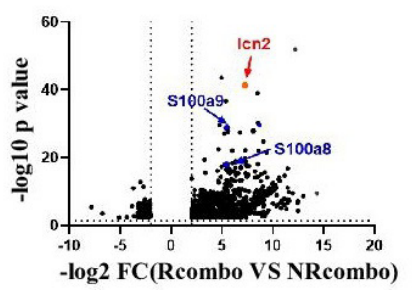

E.
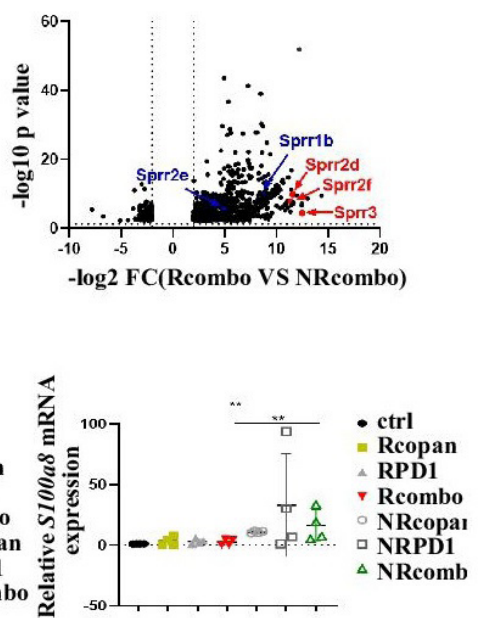

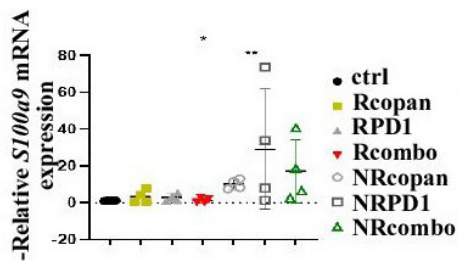

$-50 \perp, 1,1,1$,
B.

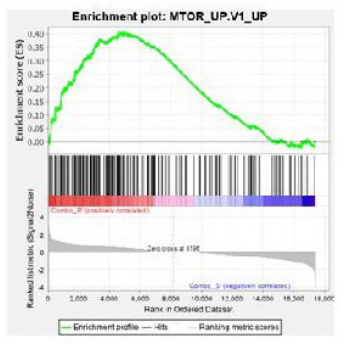

D.

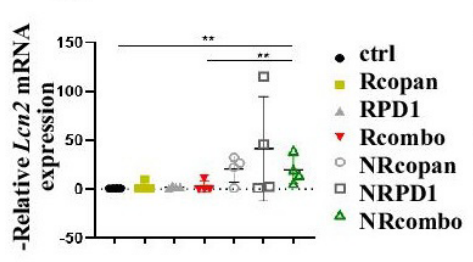

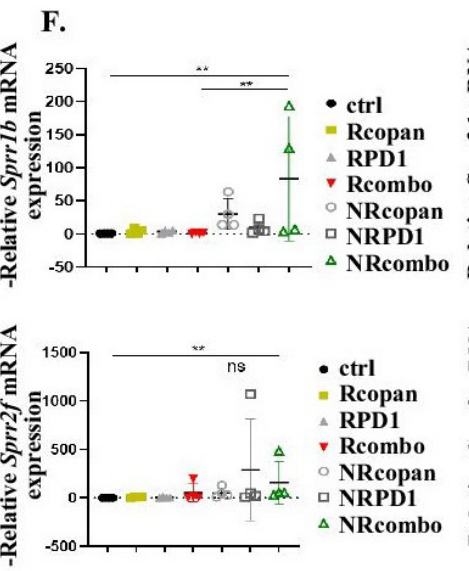
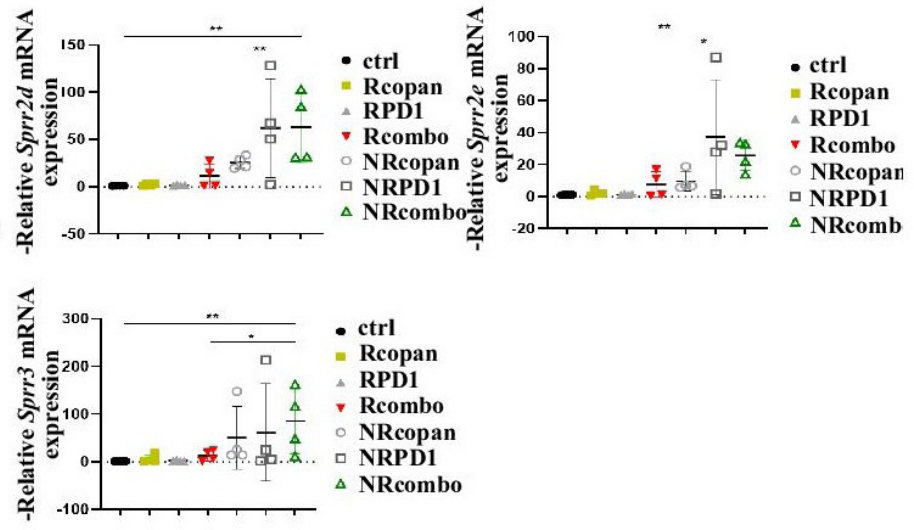

Figure 6 Alterations of the AKT/mTOR pathway and mechanisms of resistance. (A) Downregulation of the PI3K/AKT/mTOR signaling with treatment. Tumor tissues harvested at 2 weeks after treatment started were used for western blots. Left panel: copanlisib and combination therapy significantly decreased p-Akt, while aPD1 therapy did not change the p-Akt level. Right panel: quantitative analysis of the p-Akt/total Akt ration. ${ }^{* \star} p<0.01$. (B) Upregulation of the mTOR pathway with resistance: GSEA was performed on tumors harvested at 2 weeks after treatment when tumors were still responding to treatment, and tumors that were not responding to treatment. Transcriptome sequencing was performed. GSEA analysis showed that, compared with tumors sensitive to the combination therapy, upregulation of the mTOR pathway was observed in tumors not responding to the combination therapy. The NES and FDR $p$ value in the combination-resistant tumors were 1.96 and 0.002 , respectively. (C) Volcano plot showed differential gene analysis. Differential genes (RCombo vs NRcombo) from mRNA sequencing were analyzed and sorted according to - log10 p value and log2-fold change. Three genes (Lcn2, S100a8 and S100a9) along the mTOR pathway are shown. (D) Upregulation of the mTOR pathway genes in the resistant tumors. Quantitative RT-PCR of Ctrl, sensitive tumors and resistant tumors were performed. For all three genes, the combination-resistant tumors had much higher expression than the combination-sensitive ones $(p<0.01)$ and the Ctrls. (E) Upregulation of the Sprr family genes in the combination-resistant tumors. (F) Upregulation of selected Sprr genes. RT-qPCR was used to verify the mRNA expression levels of Sprr1b, Sprr2d, Sprr2e, Sprr2f, and Sprr3 in tumor tissues of each group. ${ }^{*} \mathrm{P}<0.05$, ${ }^{* *} \mathrm{P}<0.01$, ns $>0.05$ (no statistical difference). aPD1, antiprogrammed death 1; Ctrl, control; FDR, false discovery rate; GSEA, gene set enrichment analysis; mTOR, mammalian target of rapamycin; NES, normalized enrichment score; ns, not significant; p-Akt, phosphorylated Akt; PI3K, phosphoinositide 3-kinase. 
analysis was used to predict $L c n 2$-associated genes. S100A9 and $S 100 A 8$ are among the network genes strongly interacting with Lcn2 (online supplemental SI-12B).

Sprr family members were upregulated in the resistant tumors Another set of genes that is highly upregulated in the resistant tumors were several genes of the Sprr family (figure 6E). The Sprr genes encode small proline-rich proteins that are strongly induced during differentiation of human epidermal keratinocytes. There are only a few reports on the Sprr genes in UC. For example, the Sprr gene region on chromosome 1 is hypomethylated and its expression is strongly upregulated in UC $(p<0.0001){ }^{31}$ Overexpression of the Sprr genes in this family is consistently associated with inferior OS in muscle-invasive UC (online supplemental SI-13). Of the differential genes between combination-sensitive and combination-resistant tumors, Sprr2d, Sprr2f and Sprr3 were among the top 15 most highly expressed genes based on the log2-fold change (figure 6E). Of the five Sprr genes selected for RT-qPCR analysis, all five genes were consistently higher in the combination-resistant tumors when compared with the control and sensitive tumors except Sprr2f, in which the difference was not statistically different between combination-resistant and sensitive tumors (figure $6 \mathrm{~F}$ ).

\section{DISCUSSION}

The findings from this study and our previous published study ${ }^{17}$ potentially have several major impacts in the management and research of UC, 1 of the top 10 most common cancers in the USA. First, the PI3K/AKT/mTOR pathway potentially is another target for the treatment of advanced UC. Compared with less than $20 \%$ of activation alterations along the FGFR pathway which can be targeted by erdafitinib, the only FDA-approved targeted therapy in UC, $56 \%$ of UCs harbor at least one genomic alteration along the PI3K pathway (online supplemental figure S1B,C), and most of these alterations are putative drivers (figure 1A and online supplemental SI-3D). In aggregate and including prior studies, we confirmed its activity using multiple approaches, including the Cancer Dependency Map CRISPR knockout data, human and mouse cell lines, and two different PDXs with three different PI3K inhibitors. This suggests that this signaling pathway is functionally important and can possibly be targeted for the treatment of advanced UC.

Second, inhibition of the PI3K pathway potentiates immunotherapy with immune checkpoint inhibitor. For metastatic UCs, all other therapies, including chemotherapy (GC or MVAC), targeted therapy (erdafitinib) and antibody-drug conjugate (enfortumab vedotin and sacituzumab govitecan), can only provide incremental benefits and cancer recurs in almost all patients. For example, in the minority $(<20 \%)$ of patients with $\mathrm{UC}$ who had FGFR alterations and were treated with erdafitinib, even though the initial response rate was $40 \%$, only $30 \%$ of those who initially responded retained response for
12 months or longer, and almost all patients had cancer progression after 15 months into treatment. ${ }^{7}$ For the copanlisib treatment in lymphoma, the initial response rate was $60.6 \%$ with the disease control rate of $90 \%$. Only $26 \%$ patients received the treatment for over 1 year, and many of them discontinued due to cancer progression. ${ }^{3233}$ Immunotherapy had a low response rate (approximately $20 \%$ ). However, most of the responses were durable and $68 \%$ had a response for 12 months or longer. ${ }^{6}$ Our study showed that PI3K inhibition potentiates immunotherapy in two different bladder cancer models, with or without an activation alteration along the PI3K pathway. Therefore, this combination warrants a clinical trial to determine whether the preclinical findings can be translated into a clinical practice. In fact, one clinical trial combining copanlisib with an aPD1 antibody nivolumab in solid tumors is currently ongoing (clinicaltrials.gov identifier number: NCT04317105).

Third, this study performed a comprehensive analysis on how pan-PI3K inhibition modifies tumor immune microenvironment and stimulates anticancer immunity. Aberrant activation of the PI3K signaling pathway can have significant impacts on immunosuppressive TME. PTEN loss leads to activation of the PI3K signaling pathway, increases the expression of cytokines (such as VEGF, CCL2, and CXCL1) and PD-L1, and decreases T-cell infiltration in tumor. ${ }^{34}$ Furthermore, it inhibits autophagy, which plays an important role in $\mathrm{T}$ cellmediated cell death. Some other studies found that loss of PTEN or PIK3CA mutation is associated with upregulation of PD-L1 in many tumors. ${ }^{35}{ }^{36}$ Inhibition of the PI3K pathway can not only suppress tumor growth itself but also regulate cytokine production, inhibit immunosuppressor components such as tumor-associated macrophages, $\mathrm{T}_{\text {reg }}$ cells, stroma production, angiogenesis and promote T-cell infiltration. ${ }^{37}$

Another novel finding of this study is that inhibition of the PI3K pathway downregulates the TGF- $\beta$ pathway. TGF- $\beta$ is a pleiotropic cytokine involved in promoting tumor fibrosis, epithelial-mesenchymal transition, tumor angiogenesis and suppression of immune response. ${ }^{38} 39$ It can mitigate the cytotoxic effects of CD8 T cells and decrease granzyme expression, ${ }^{40}$ upregulate FOXP3, the master transcription regulator of $\mathrm{T}_{\text {reg }}$ cells, ${ }^{41}$ affect $\mathrm{MHC}$ gene expression and antigen presentation, ${ }^{42}$ and stimulate other immunosuppressive cells. In bladder cancer, TGF- $\beta$ attenuates tumor response to immunotherapy by excluding T-cell infiltration into tumors. ${ }^{43}$ In this study, the downregulation of TGF- $\beta$ was observed in the copanlisib treatment, but not in the aPD1 or combination groups. It is possible that the aPD1 therapy overrides copanlisib in its suppression of the TGF- $\beta$ pathway in the combination group. In addition to its effect on TGF $\beta$ downregulation, copanlisib induced CD8 T-cell infiltration, decreased $\mathrm{T}_{\text {reg }}$-cell infiltration, increased CD80, CD86, MHC-I and MHC-II expression on DCs, upregulated immune activation markers, and increased the expression of the antigen processing and presentation 
machinery. In summary, pan-PI3K inhibition can possibly potentiate immunotherapy through regulating multiple pathways in the immune system.

Fourth, this study sheds insight to the resistance mechanisms of targeted immunotherapy. Secondary resistance is the most common cause of treatment failure after initial response. Two major mechanisms have been proposed, adaptive resistance and acquired resistance. For acquired resistance, acquirement of a new genomic alteration or outgrowth of resistant subclones has been described in other targeted therapies as seen with the epidermal growth factor receptor gene T790M mutation in non-small cell lung cancer. ${ }^{44}$ We previously found no new acquired mutation or enrichment of subclonal mutations of a UC PDXs carrying a PIK3CA H1047R after prolonged treatment and development of secondary resistance to a PI3K inhibitor pictilisib. ${ }^{17}$ For adaptive resistance, there are numerous upstream receptors of the PI3K pathway, and extensive crosstalk among the PI3K pathway and other pathways. PI3K isoform switch can also contribute to reactivation of the PI3K pathway. ${ }^{45}$ Our studies suggest that these adaptive mechanisms are the main mechanisms of secondary resistance based on our PDX and cell line studies. In our previous PDX studies, we observed increase of p-AKT in both PDXs, one with an activation mutation and the other one with PIK3CA gene amplification. ${ }^{17}$ Here we showed that the mTOR pathway was upregulated per GSEA analysis, which was further validated with RT-qPCR of a few selected genes (figure 6).

Our findings of Lcn2 and Sprr family genes in the secondary resistance to PI3K inhibition are novel (figure 6). LCN2 is a secreted glycoprotein that transports small lipophilic ligands ${ }^{46}$ and a downstream factor of the PI3K/Akt signaling. High LCN2 expression enhances cancer cell proliferation, migration and invasion. ${ }^{47}$ LCN2 is involved in mediating immunity tolerance by promoting the infiltration of $\mathrm{T}_{\text {reg }}$ cells. Moreover, LCN2 is involved in the progression of tumors through regulating MMP family proteins and promoting tumor cell invasion and migration. ${ }^{48}$ S100A9 and S100A8 play important roles in inflammation and cancer. ${ }^{49-51}$ By comparative analysis of the differential genes between responders and NRs, it was found that all these genes were highly expressed in the NR group, which was further confirmed with quantitative RT-qPCR. GeneMANIA interaction network analysis showed all these genes are highly integrated (online supplemental SI-12). The crosstalk of these genes may not only contribute to resistance to PI3K inhibition but may also lead to escape of anticancer immunity.

Another important finding is the involvement of the Sprr family genes in the resistance to the combination therapy. The SPRR family proteins are closely related to the epithelial cell development and tumor progression. $^{52}$ There are several reports on the involvement of $S P R R$ genes in progression of other cancers. ${ }^{53} 54$ The only report of its involvement in UC is its overexpression in cancer-initiating cells and squamous differentiation. ${ }^{55}$ Our data showed the upregulation of multiple members of its family (figure 6E,F) and the association of high expression with poor prognosis across multiple genes of this family in UC (online supplemental SI-13), suggesting that further investigation of the involvement of this family of genes in bladder cancer is warranted.

There are several limitations associated with this study. First, only two bladder cancer models were tested in this study. It is not clear whether the findings in these two models are applicable to other models. Second, it is not clear whether the findings in mouse models are applicable in human patients. Third, it is not clear how inhibition of PI3K affects T-cell transcriptional program. Much more studies are needed to further elucidate the mechanism.

\section{CONCLUSIONS}

Over $50 \%$ of advanced UCs harbor genomic alterations along the PI3K/AKT/mTOR pathway. Inhibition of the PI3K pathway inhibits cell growth, migration and colony forming in cell lines carrying activation alterations along this pathway. A pan-PI3K inhibitor potentiates aPD1/ PD-L1 immunotherapy in a syngeneic mouse model from a cell line carrying Pten deletion and a cell line without activation alteration along the PI3K pathway. This combination treatment is associated with downregulation of the TGF- $\beta$ pathway, upregulation of the antigen processing and presentation machinery, increased infiltration of CD8 $\mathrm{T}$ cells, decreased $\mathrm{T}_{\text {reg }}$ cells, increased expression of costimulatory immune molecules, stimulation of DC functions, and increased expression of immunostimulatory cytokines at the TME. Secondary resistance is associated with the reactivation of the AKT/mTOR pathway and Sprr family genes. In conclusion, a clinical trial to determine the efficacy and toxicity of the combination of a pan-PI3K inhibitor and immune checkpoint inhibitor in bladder cancer is warranted to determine the efficacy and toxicity of this combination in clinical patients.

\section{Author affiliations}

${ }^{1}$ Department of Internal Medicine, University of California Davis, Sacramento, CA, USA

${ }^{2}$ Department of Urology, Renmin Hospital of Wuhan University, Wuhan, People's Republic of China

${ }^{3}$ Department of Biochemistry and Molecular Medicine, University of California Davis, Sacramento, CA, USA

${ }^{4}$ Department of Medicine, Harvard Medical School, Boston, MA, USA

${ }^{5}$ Department of Oncology, Renmin Hospital of Wuhan University, Wuhan, People's Republic of China

${ }^{6}$ Department of Bioengienering, Massachusetts Institute of Technology, Cambridge, MA, USA

${ }^{7}$ Dana-Farber Cancer Institute, Harvard Medical School, Boston, MA, USA ${ }^{8}$ Department of Urology, VA Boston Healthcare System, Boston, MA, USA ${ }^{9}$ Brigham and Women's Hospital, Harvard Medical School, Boston, MA, USA

${ }^{10}$ Department of Medicine, VA Boston Healthcare System, Boston, MA, USA

\section{Twitter Guru Sonpavde @sonpavde}

Acknowledgements The authors thank William Kim, MD, PhD, from University of North Carolina, for providing cell lines UPPL1541 and BBN963 for this study.

Contributors SZ, A-HM and ZZ designed and performed the experiments, analyzed the data, and contributed to writing the paper. EA and DK performed the bioinformatic analysis. TR, NL, KN, VCSRC, RP and JGR provided essential 
experimental tools and support. KM and GS contributed to project conception, data analysis, manuscript preparation and final approval. FC was the mentor and cosupervised SZ's research. C-xP designed the investigation, analyzed the data, supervised the study, and wrote the paper. All authors read and approved the manuscript as submitted. C-xP is responsible for the overall content of this manuscript.

Funding Funding work was supported in part by U54 Grant (grant number 1 U54 CA233306; C-xPprincipal investigator (PI): C-xP), R01 Grant (grant number 1R01CA176803-01; PI: C-XP), and Merit Review (award number 1101 BX003840, PI: C-xP) from the US Department of Veterans Affairs Biomedical Laboratory Research and Development Program. The contents do not represent the views of the US Department of Veterans Affairs or the US government.

Competing interests There are no competing interests associated with this project. The following investigators received support that is not related to this project: GS: advisory board: BMS, Genentech, EMD Serono, Merck, Sanofi, Seattle Genetics/Astellas, AstraZeneca, Exelixis, Janssen, Bicycle Therapeutics, Pfizer, Immunomedics/Gilead, Scholar Rock, and G1 Therapeutics; research support to institution: Sanofi, AstraZeneca, Immunomedics/Gilead, QED, Predicine, and BMS; steering committee of studies: BMS, Bavarian Nordic, Seattle Genetics, QED, G1 Therapeutics (all unpaid), and AstraZeneca, EMD Serono, Debiopharm (paid); data safety monitoring committee: Mereo; travel costs: BMS (2019) and AstraZeneca (2018); writing/editor fees: Uptodate; editor of Elsevier Practice Update Bladder Cancer Center of Excellence; speaking fees: Physicians Education Resource, Onclive, Research to Practice, and Medscape (all educational). DK: Research contracts: Revolution Medicines, Genentech; consultant: AADi.

Patient consent for publication Not required.

Provenance and peer review Not commissioned; externally peer reviewed.

Data availability statement Data are available in a public, open access repository. Data are available upon reasonable request. All data relevant to the study are included in the article or uploaded as supplementary information.

Supplemental material This content has been supplied by the author(s). It has not been vetted by BMJ Publishing Group Limited (BMJ) and may not have been peer-reviewed. Any opinions or recommendations discussed are solely those of the author(s) and are not endorsed by BMJ. BMJ disclaims all liability and responsibility arising from any reliance placed on the content. Where the content includes any translated material, BMJ does not warrant the accuracy and reliability of the translations (including but not limited to local regulations, clinical guidelines, terminology, drug names and drug dosages), and is not responsible for any error and/or omissions arising from translation and adaptation or otherwise.

Open access This is an open access article distributed in accordance with the Creative Commons Attribution Non Commercial (CC BY-NC 4.0) license, which permits others to distribute, remix, adapt, build upon this work non-commercially, and license their derivative works on different terms, provided the original work is properly cited, appropriate credit is given, any changes made indicated, and the use is non-commercial. See http://creativecommons.org/licenses/by-nc/4.0/.

\section{ORCID iDs}

Elio Adib http://orcid.org/0000-0003-0594-7976

Guru Sonpavde http://orcid.org/0000-0002-1010-9611

Chong-xian Pan http://orcid.org/0000-0002-8588-3994

\section{REFERENCES}

1 Bray F, Ferlay J, Soerjomataram I, et al. Global cancer statistics 2018: GLOBOCAN estimates of incidence and mortality worldwide for 36 cancers in 185 countries. CA Cancer J Clin 2018;68:394-424.

2 Ferlay J, Colombet M, Soerjomataram I, et al. Estimating the global cancer incidence and mortality in 2018: GLOBOCAN sources and methods. Int J Cancer 2019;144:1941-53.

3 Siegel RL, Miller KD, Fuchs HE, et al. Cancer statistics, 2021. CA Cancer J Clin 2021;71:7-33.

4 von der Maase H, Hansen SW, Roberts JT, et al. Gemcitabine and cisplatin versus methotrexate, vinblastine, doxorubicin, and cisplatin in advanced or metastatic bladder cancer: results of a large, randomized, multinational, multicenter, phase III study. J Clin Oncol 2000;18:3068-77.

5 Powles T, Park SH, Voog E, et al. Avelumab maintenance therapy for advanced or metastatic urothelial carcinoma. N Engl J Med 2020;383:1218-30.
6 Bellmunt J, de Wit R, Vaughn DJ, et al. Pembrolizumab as secondline therapy for advanced urothelial carcinoma. $N$ Engl J Med 2017;376:1015-26.

7 Loriot Y, Necchi A, Park SH, et al. Erdafitinib in locally advanced or metastatic urothelial carcinoma. N Engl J Med 2019;381:338-48.

8 Powles T, Rosenberg JE, Sonpavde GP, et al. Enfortumab vedotin in previously treated advanced urothelial carcinoma. $N$ Engl $J$ Med2021.

9 Tagawa ST, Balar AV, Petrylak DP, et al. TROPHY-U-01: a phase II open-label study of Sacituzumab Govitecan in patients with metastatic urothelial carcinoma progressing after platinumbased chemotherapy and checkpoint inhibitors. J Clin Oncol 2021;39:JCO2003489.

10 Robertson AG, Kim J, Al-Ahmadie H, et al. Comprehensive molecular characterization of muscle-invasive bladder cancer. Cell 2018;174:1033.

11 Guerrero-Zotano A, Mayer IA, Arteaga CL. Pi3K/AKT/mTOR: role in breast cancer progression, drug resistance, and treatment. Cancer Metastasis Rev 2016;35:515-24.

12 Aragoneses-Fenoll L, Ojeda G, Montes-Casado M, et al. T-cellspecific loss of the Pl-3-kinase $\mathrm{p} 110 \alpha$ catalytic subunit results in enhanced cytokine production and antitumor response. Front Immunol 2018;9:332.

13 Abu Eid R, Ahmad S, Lin Y, et al. Enhanced therapeutic efficacy and memory of tumor-specific CD8 T cells by Ex Vivo PI3K- $\delta$ inhibition. Cancer Res 2017;77:4135-45.

14 Aksoy E, Saveanu L, Manoury B. The isoform selective roles of PI3Ks in dendritic cell biology and function. Front Immunol 2018;9:2574.

15 Liu N, Rowley BR, Bull CO, et al. BAY 80-6946 is a highly selective intravenous PI3K inhibitor with potent $\mathrm{p} 110 \alpha$ and $\mathrm{p} 110 \delta$ activities in tumor cell lines and xenograft models. Mol Cancer Ther 2013;12:2319-30.

16 Saito R, Smith CC, Utsumi T, et al. Molecular subtype-specific immunocompetent models of high-grade urothelial carcinoma reveal differential neoantigen expression and response to immunotherapy. Cancer Res 2018;78:3954-68.

17 Zeng S-X, Zhu Y, Ma A-H, et al. The phosphatidylinositol 3-kinase pathway as a potential therapeutic target in bladder cancer. Clin Cancer Res 2017;23:6580-91.

18 Tsherniak A, Vazquez F, Montgomery PG, et al. Defining a cancer dependency MAP. Cell 2017;170:564-76.

19 Pan C-X, Zhang H, Tepper CG, et al. Development and characterization of bladder cancer patient-derived xenografts for molecularly guided targeted therapy. PLoS One 2015;10:e0134346.

20 Nakagawa T, Zhu H, Morishima N, et al. Caspase-12 mediates endoplasmic-reticulum-specific apoptosis and cytotoxicity by amyloid-beta. Nature 2000;403:98-103.

21 Kaufman RJ. Stress signaling from the lumen of the endoplasmic reticulum: coordination of gene transcriptional and translational controls. Genes Dev 1999;13:1211-33.

22 Cao J, Li J, Yang X, et al. Transcriptomics analysis for the identification of potential age-related genes and cells associated with three major urogenital cancers. Sci Rep 2021;11:641.

23 Saleh R, Elkord E. FoxP3 ${ }^{+} \mathrm{T}$ regulatory cells in cancer: Prognostic biomarkers and therapeutic targets. Cancer Lett 2020;490:174-85.

24 Holmgaard RB, Zamarin D, Li Y, et al. Tumor-expressed IDO recruits and activates MDSCs in a Treg-Dependent manner. Cell Rep 2015;13:412-24.

25 De Henau O, Rausch M, Winkler D, et al. Overcoming resistance to checkpoint blockade therapy by targeting $\mathrm{PI} 3 \mathrm{~K} \gamma$ in myeloid cells. Nature 2016;539:443-7.

26 Zitvogel L, Galluzzi L, Kepp O, et al. Type I interferons in anticancer immunity. Nat Rev Immunol 2015;15:405-14.

27 Zhong X, Xie F, Chen L, et al. S100A8 and S100A9 promote endothelial cell activation through the RAGE-mediated mammalian target of rapamycin complex 2 pathway. Mol Med Rep 2020;22:5293-303.

28 Xiao X, Yeoh BS, Vijay-Kumar M. Lipocalin 2: an emerging player in iron homeostasis and inflammation. Annu Rev Nutr 2017;37:103-30.

29 Gomez-Chou SB, Swidnicka-Siergiejko AK, Badi N, et al. Lipocalin-2 promotes pancreatic ductal adenocarcinoma by regulating inflammation in the tumor microenvironment. Cancer Res 2017;77:2647-60.

30 Wang Y-P, Yu G-R, Lee M-J, et al. Lipocalin-2 negatively modulates the epithelial-to-mesenchymal transition in hepatocellular carcinoma through the epidermal growth factor (TGF-beta1)/Lcn2/Twist1 pathway. Hepatology 2013;58:1349-61.

31 Reinert T, Modin C, Castano FM, et al. Comprehensive genome methylation analysis in bladder cancer: identification and validation of novel methylated genes and application of these as urinary tumor markers. Clin Cancer Res 2011;17:5582-92. 
32 Dreyling M, Morschhauser F, Bouabdallah K, et al. Phase II study of copanlisib, a PI3K inhibitor, in relapsed or refractory, indolent or aggressive lymphoma. Ann Oncol 2017;28:2169-78.

33 Dreyling M, Santoro A, Mollica L, et al. Long-term safety and efficacy of the PI3K inhibitor copanlisib in patients with relapsed or refractory indolent lymphoma: 2-year follow-up of the CHRONOS-1 study. Am $J$ Hematol2019.

34 Peng W, Chen JQ, Liu C, et al. Loss of PTEN promotes resistance to T cell-mediated immunotherapy. Cancer Discov 2016;6:202-16.

35 Kobayashi Y, Lim S-O, Yamaguchi H. Oncogenic signaling pathways associated with immune evasion and resistance to immune checkpoint inhibitors in cancer. Semin Cancer Biol 2020;65:51-64.

36 Crane CA, Panner A, Murray JC, et al. $\mathrm{PI}(3)$ kinase is associated with a mechanism of immunoresistance in breast and prostate cancer. Oncogene 2009;28:306-12.

37 O'Donnell JS, Massi D, Teng MWL, et al. Pi3K-AKT-mTOR inhibition in cancer immunotherapy, redux. Semin Cancer Biol 2018;48:91-103.

38 Batlle E, Massagué J. Transforming growth factor- $\beta$ signaling in immunity and cancer. Immunity 2019;50:924-40.

39 Ciardiello D, Elez E, Tabernero J, et al. Clinical development of therapies targeting TGF $\beta$ : current knowledge and future perspectives. Ann Oncol 2020;31:1336-49.

40 Thomas DA, Massagué J. TGF-beta directly targets cytotoxic T cell functions during tumor evasion of immune surveillance. Cancer Cell 2005;8:369-80.

41 Chen M-L, Pittet MJ, Gorelik L, et al. Regulatory T cells suppress tumor-specific CD8 T cell cytotoxicity through TGF-beta signals in vivo. Proc Natl Acad Sci U S A 2005;102:419-24.

42 Nandan D, Reiner NE. TGF-beta attenuates the class II transactivator and reveals an accessory pathway of IFN-gamma action. J Immunol 1997;158:1095-101.

43 Mariathasan S, Turley SJ, Nickles D, et al. TGFbeta attenuates tumour response to PD-L1 blockade by contributing to exclusion of $\mathrm{T}$ cells. Nature 2018;554:544-8.
44 Kobayashi S, Boggon TJ, Dayaram T, et al. EGFR mutation and resistance of non-small-cell lung cancer to gefitinib. $N$ Engl J Med 2005;352:786-92.

45 Fruman DA, Chiu H, Hopkins BD, et al. The PI3K pathway in human disease. Cell 2017;170:605-35.

46 Hu C, Yang K, Li M, et al. Lipocalin 2: a potential therapeutic target for breast cancer metastasis. Onco Targets Ther 2018;11:8099-106.

47 Yang J, Moses MA. Lipocalin 2: a multifaceted modulator of human cancer. Cell Cycle 2009;8:2347-52.

48 Santiago-Sánchez GS, Pita-Grisanti V, Quiñones-Díaz B, et al. Biological functions and therapeutic potential of lipocalin 2 in cancer. Int J Mol Sci 2020;21 doi:10.3390/ijms21124365

49 Vogl T, Stratis A, Wixler V, et al. Autoinhibitory regulation of S100A8/ S100A9 alarmin activity locally restricts sterile inflammation. J Clin Invest 2018;128:1852-66.

50 Feng $\mathrm{P}-\mathrm{H}$, Chen K-Y, Huang Y-C, et al. Bevacizumab reduces S100A9-positive MDSCs linked to intracranial control in patients with EGFR-mutant lung adenocarcinoma. J Thorac Oncol 2018;13:958-67.

51 Ohata H, Shiokawa D, Obata Y, et al. Nox1-dependent mTORC1 activation via S100A9 oxidation in cancer stem-like cells leads to colon cancer progression. Cell Rep 2019;28:1282-95.

52 Carregaro F, Stefanini ACB, Henrique T, et al. Study of small prolinerich proteins (SPRRs) in health and disease: a review of the literature. Arch Dermatol Res 2013;305:857-66.

53 Fang Z, Zhang S, Wang Y, et al. Long non-coding RNA MALAT-1 modulates metastatic potential of tongue squamous cell carcinomas partially through the regulation of small proline rich proteins. BMC Cancer 2016;16:706.

$54 \mathrm{Kim} \mathrm{JC}, \mathrm{Yu} \mathrm{JH}$, Cho YK, et al. Expression of SPRR3 is associated with tumor cell proliferation in less advanced stages of breast cancer. Breast Cancer Res Treat 2012;133:909-16.

55 Hoggarth ZE, Osowski DB, Slusser-Nore A, et al. Enrichment of genes associated with squamous differentiation in cancer initiating cells isolated from urothelial cells transformed by the environmental toxicant arsenite. Toxicol Appl Pharmacol 2019;374:41-52. 